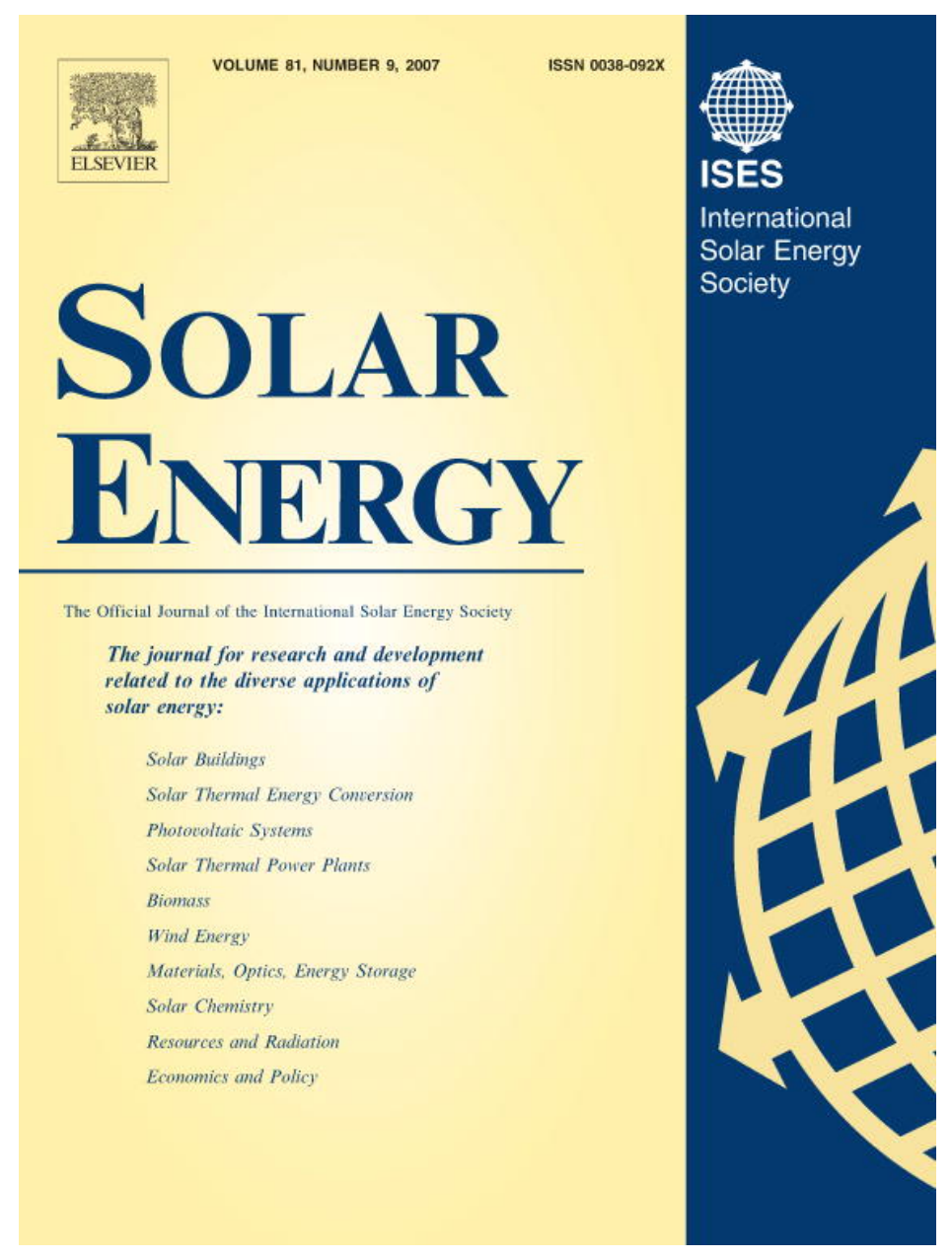

This article was published in an Elsevier journal. The attached copy

is furnished to the author for non-commercial research and education use, including for instruction at the author's institution, sharing with colleagues and providing to institution administration.

Other uses, including reproduction and distribution, or selling or licensing copies, or posting to personal, institutional or third party websites are prohibited.

In most cases authors are permitted to post their version of the article (e.g. in Word or Tex form) to their personal website or institutional repository. Authors requiring further information regarding Elsevier's archiving and manuscript policies are encouraged to visit: 


\title{
Quantum dot containing nanocomposite thin films for photoluminescent solar concentrators
}

\author{
A. Schüler ${ }^{\mathrm{a}, *}$, M. Python ${ }^{\mathrm{b}}$, M. Valle del Olmo ${ }^{\text {a }}$, E. de Chambrier ${ }^{\text {a }}$ \\ ${ }^{a}$ Solar Energy Laboratory LESO-PB, Ecole Polytechnique Fédérale de Lausanne EPFL, Switzerland \\ ${ }^{\mathrm{b}}$ Institute of Microtechnology IMT, University of Neuchâtel, CH-2000 Neuchâtel, Switzerland \\ Received 28 August 2006; received in revised form 10 January 2007; accepted 17 January 2007 \\ Available online 27 February 2007 \\ Communicated by: Associate Editor Dr. D. Yogi Goswami
}

\begin{abstract}
Silicon oxide films containing CdS quantum dots have been deposited on glass substrates by a sol-gel dip-coating process. Hereby the CdS nanocrystals are grown during the thermal annealing step following the dip-coating procedure. Total hemispherical transmittance and reflectance measurements were carried out by means of a spectrophotometer coupled to an integrating sphere. For CdS-rich films, an absorption edge at photon energies in the vicinity of the band gap value of bulk CdS is observed. For lower CdS concentrations, the absorption edge shifts to higher photon energies, as expected for increasing quantum confinement. The samples show visible photoluminescence which is concentrated by total internal reflection and emitted at the edges of the substrate. The edge emission has been characterized by angle-dependent photoluminescent (PL) spectroscopy. Information on the lateral energy transport within the sample can be extracted from spectra obtained under spatial variation of the spot of excitation. The color of the photoluminescence can be tuned by varying the annealing temperature which governs crystal growth and thus the cluster size distribution. The characteristic features observed in the PL spectra clearly exhibit a blueshift for lower annealing temperatures, confirming the presence of quantum size effects.

Advantages of the proposed concept of quantum dot containing coatings on glass panes for photoluminescent solar concentrators are the high potential for low-cost fabrication on the large scale and the suitability for architectural integration.
\end{abstract}

(c) 2007 Elsevier Ltd. All rights reserved.

Keywords: Fluorescent planar solar concentrators; Quantum dots; CdS nanocrystals; Quantum size effects; Nanocomposite materials; Sol-gel dip-coating; Thin film technology; Photovoltaic cells

\section{Introduction}

Due to their fascinating optical and electronic properties, semiconductor nanocrystals have attracted considerable attention in the recent years (see e.g. Nirmal et al., 1996). The three-dimensional quantum confinement in the so-called quantum dots induces a discretization of electronic states, very much like in atoms or molecules. The resulting optical properties depend on the crystal size and can thus be tuned by varying the dimensions of the nano-

\footnotetext{
${ }^{*}$ Corresponding author. Tel.: +41 216934544.

E-mail address: andreas.schueler@epfl.ch (A. Schüler).
}

crystals. Quantum dots are considered as promising candidates for optoelectronic applications including lightemitting diodes (Tasco et al., 2004) and quantum dot lasers (Darugar et al., 2006). Various concepts have been proposed for the use of nanostructured materials in solar energy conversion (Oelhafen and Schüler, 2005). Quantum dots might be used as frequency converters to better match the spectrum of the incoming radiation to the spectral efficiency of the solar cell (Van Sark et al., 2005). Semiconductor nanostructures open the way to intermediate band solar cells with high efficiencies (Luque and Martí, 1997). By introducing zero dimensional structures into photovoltaic cells, the creation of multiple electron-hole pairs per 
photon through impact ionization can be achieved, suggesting a potential capability of quantum yields $>1$ (Nozik, 2002). In quantum dot sensitized nanocrystalline $\mathrm{TiO}_{2}$ solar cells, semiconductor nanocrystals substitute for organic molecules (Plass et al., 2002; Wijayantha et al., 2004). Hereby the possible advantages of quantum dots over dye molecules include the tunability of optical properties with size and better heterojunction formation with solid hole conductors. Photovoltaic effects have been reported for quantum dots immersed in hole-conducting MEH-PPV (Greenham et al., 1996), and it has been proposed to use a blend of electron and hole conducting polymers as host matrix (Nozik, 2002). One of the most promising applications might be planar photoluminescent concentrators for photovoltaics where high concentration factors on the solar cells can be achieved even for diffuse solar radiation. Such devices have originally been designed on the basis of organic dyes (Goetzberger and Greubel, 1977), and might benefit from a considerably improved lifetime when replacing the organic fluorescent substances by inorganic quantum dots (Barnham et al., 2000; Gallagher et al., 2004). Additionally, the tunability of the optical properties by the size of the nanocrystals provide a large amount freedom for the design and optimization of such devices.

The fabrication of nanometer-sized crystallites can be achieved by growth in colloidal solutions (Murray et al., 1993; Crouch et al., 2003), on surfaces in vacuum deposition processes (Lee et al., 2006; Schüler et al., 1999, 2001), by electron beam lithography (Ishikawa et al., 2000), or by sol-gel techniques (Parvathy et al., 1997; Lifshitz et al., 1998). Often core-shell structures are used to passivate the surfaces of the nanocrystals (e.g. CdSe core in $\mathrm{ZnS}$ shell). For colloidal quantum dots, strong and tuneable visible photoluminescence has been observed with quantum yields in the range from $50 \%$ to $85 \%$ (Hines and Guyot-Sionnest, 1996; De Mello Donegá et al., 2003).

In this paper we propose to fabricate fluorescent solar concentrators by coating transparent substrates with quantum dot containing nanocomposite thin films. We use the sol-gel dip-coating process for the deposition of photoluminescent CdS nanocrystal containing silica films. The absorptance and the photoluminescence of the prepared samples are subsequently characterized by optical spectroscopies.

\section{Theory}

Éfros and Éfros (1982) pointed out that, when the dimensions of the semiconductor nanocrystal become smaller than the effective Bohr radius $a_{\mathrm{B}}^{*}$, the electron and the hole should occupy primarily the individual lowest eigenstate of the quantum well with relatively little spatial correlation (regime of the individual particle confinement). Brus proposed a simple one electron-hole pair (exciton) model of semiconductor nanocrystals, using an effective mass approximation based on a parabolic band (Brus,
1983). In the limit of strong confinement, the following expression can be derived for the lowest excited state energy $E$ (Kayanuma, 1988):

$E=E_{\mathrm{g}}+\frac{\hbar^{2} \pi^{2}}{2 R^{2}}\left[\frac{1}{m_{\mathrm{e}}} \frac{1}{m_{\mathrm{h}}}\right]-\frac{1.786 e^{2}}{\varepsilon R}-0.248 E_{\mathrm{Ry}}^{*}$,

with the effective Rydberg energy $E_{\mathrm{Ry}}^{*}$

$E_{\mathrm{Ry}}^{*}=e^{4} / 2 \varepsilon^{2} \hbar^{2}\left(\frac{1}{m_{\mathrm{e}}}+\frac{1}{m_{\mathrm{h}}}\right)$.

Here $E_{\mathrm{g}}$ designates the $0 \mathrm{~K}$ bulk band gap, $R$ the crystallite radius, $m_{\mathrm{e}(\mathrm{h})}$ the effective mass of the electron (hole), and $\varepsilon$ the dielectric constant. Two major physical effects affect the lowest excited state energy, the contribution of the spatial localization of electron and hole varying as $R^{-2}$, and the size dependent Coulomb attraction varying as $R^{-1}$. For small $R$ the localization term dominates and $E$ is shifted to higher energies than $E_{\mathrm{g}}$. As $E$ shifts to higher energies, its oscillator strength increases as the spatial overlap of the electron and hole wavefunctions increases. The last term in Eq. (1) including the effective Rydberg energy is the remnant of the exciton effect.

The above described model has been modified and extended by other authors concerning the existence of a bound state of two electron-hole pairs within a single dot (biexciton) ( $\mathrm{Hu}$ et al., 1990), and confinement-induced band mixing (Kang et al., 1992).

Using a finite depth well model, Nosaka calculated the energy shift of the lowest excited state for ultrasmall CdS particles (Nosaka, 1991). Assuming effective masses of electron and hole $m_{\mathrm{e}}=0.19$ and $m_{\mathrm{h}}=0.80$, the dielectric constant $\varepsilon=5.6$, and the potential depth $V_{0}=3.6 \mathrm{eV}$, energy shifts of $0.4 \mathrm{eV}$ and $1.2 \mathrm{eV}$ are predicted at cluster radii of $2 \mathrm{~nm}$ and $1 \mathrm{~nm}$, respectively. The energy shifts calculated from Nosaka's finite depth well model are in general somewhat lower than the values predicted by Eq. (1) derived for infinitely deep potential wells. This reflects the fact that in the finite depth model, the wavefunction leaks out to some extent, implying a weaker quantum confinement. Tight binding calculations (Lippens and Lannoo, 1989) and experimental data (Wang and Herron, 1990) yield energy shifts in the same order of magnitude as those obtained by Nosaka's finite depth well model.

\section{Experimental}

A sketch of the principle of the original proposition (Goetzberger and Greubel, 1977) of fluorescent concentrators in shown in Fig. 1a. One or several waveguides are fabricated from panes of transparent media doped with fluorescent dyes. Incoming solar radiation is absorbed in the volume of the waveguides, and isotropically re-emitted by fluorescence. A large part of the emitted radiation is captured by total internal reflection and propagates to the edges. The likewise concentrated radiation is converted 

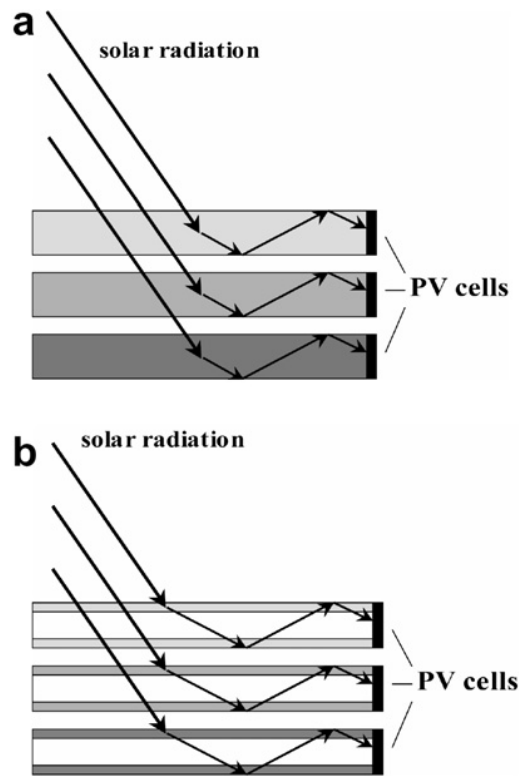

Fig. 1. (a) Schematic drawing of the principle of fluorescent solar concentrators proposed in Goetzberger and Greubel (1977). The incoming solar radiation is absorbed in the bulk material. The re-emitted radiation is concentrated by total internal reflection within the waveguides. In stacked fluorescent concentrators, the absorption edges of the dyes are chosen in a manner similar to multi-junction photovoltaic cells. (b) When a fluorescent coating is deposited on transparent substrate, the total internal reflection will occur mainly at the surface of the coating. The coating can be made of a quantum dot containing nanocomposite material, the substrate can consist of e.g. a highly transparent low iron solar glass. For clarity, the thickness of the coating is exaggerated in the representation.

by photovoltaic cells with band gaps matching the wavelengths of the spectral emission lines of the photoluminescent materials. By choosing dyes with suitable absorption and emission properties, stacks of fluorescent collectors can be designed, with absorption edges chosen in a manner similar to multi-junction photovoltaic cells. The same geometry has been used in quantum dot solar concentrators (Barnham et al., 2000; Gallagher et al., 2004), where photoluminescent semiconductor nanocrystals replace the fluorescent dyes. Instead of immersing quantum dots in transparent resins, we deposit CdS quantum dot containing silicon dioxide films $\left(\mathrm{SiO}_{2}: \mathrm{CdS}\right)$ on highly transparent low iron glass substrates by sol-gel dip-coating. If the refractive index of the coating is close to the one of the substrate, internal reflection occurs mainly at the surface of the coating, as depicted in Fig. 1b. For clarity, the thickness of the coating is exaggerated in the representation.

The dip-coating solutions are prepared in a laminar flux chapel on the basis of tetraethyl orthosilicate (TEOS, $\left.\mathrm{C}_{8} \mathrm{H}_{20} \mathrm{O}_{4} \mathrm{Si}\right)$, cadmium acetate dihydrate $\left(\mathrm{C}_{4} \mathrm{H}_{6} \mathrm{O}_{4}\right.$ $\left.\mathrm{Cd} \cdot 2 \mathrm{H}_{2} \mathrm{O}\right)$ and thiourea $\left(\mathrm{CH}_{4} \mathrm{~N}_{2} \mathrm{~S}\right)$, as precursors for silicon, cadmium and sulphur, respectively. Tetraethyl orthosilicate (TEOS, $\mathrm{C}_{8} \mathrm{H}_{20} \mathrm{O}_{4} \mathrm{Si}$ ) is hydrolyzed in presence of nitric acid $\mathrm{HNO}_{3}$ and water $\mathrm{H}_{2} \mathrm{O}$. During hydrolysis, the solution is stirred and heated to $50{ }^{\circ} \mathrm{C}$ for a period of sev- eral hours. Triton-x-100 is added in order to improve the wettability in the dip-coating process. A mixture of cadmium acetate dihydrate $\left(\mathrm{C}_{4} \mathrm{H}_{6} \mathrm{O}_{4} \mathrm{Cd} \cdot 2 \mathrm{H}_{2} \mathrm{O}\right)$ and thiourea $\left(\mathrm{CH}_{4} \mathrm{~N}_{2} \mathrm{~S}\right)$ based solutions is added to the TEOS based solution after hydrolysis of the latter.

A suitable apparatus provides a vibration-free and regular movement for the dipping and withdrawal of the substrates. A servo drive (MKD 25B REXROTH) is controlled by a feedback loop (control unit Ecodrive 03 REXROTH INDRAMAT). The speed of the rotation is adapted by a Neugart transmission and transformed into a translational movement by a helix equipped with a special ball bearing (compact module CKK 12-90, REXROTH). A microcomputer is used to command the control unit of the servo drive. For dust-protection the dip-coater is placed in a laminar flux chapel (SKANAIR VFC 120). The air quality is monitored by real-time particle counting (KLOTZ PSSair with isocinetic nozzle). In the laminar flux chapel typically less than 1 particle larger than $0.3 \mu \mathrm{m}$ is counted per cubic foot (for comparison: ambient laboratory air: $\sim 10^{5}$ particles per cubic ft). After dip-coating, the resulting thin xerogel films are tempered at $250-450{ }^{\circ} \mathrm{C}$ in nitrogen atmosphere. During thermal annealing, samples are enclosed in special containers to avoid dust contamination during sample transfer and tempering. Before dip-coating, substrates are cleaned thoroughly in an ultrasonic bath (BRANSON 8510). In this study, highly transparent white glass substrates of the dimensions $76 \mathrm{~mm} \times 26 \mathrm{~mm} \times 1 \mathrm{~mm}$ have been used.

Spectral measurements of the photoluminescence have been performed in the VIS range ( $380-820 \mathrm{~nm}$ ) by a grating spectrophotometer (ORIEL MS $125^{\mathrm{TM}}$ 1/8m Spectrograph, with Instaspec ${ }^{\mathrm{TM}}$ II Photodiode Array Detector and sighting optics). An argon/mercury discharge lamp serves as wavelength standard for the calibration of the spectrophotometer. Samples are excited by broad band ultraviolet radiation with wavelengths in the order of $365 \mathrm{~nm}$ (UVA). The concentrated visible light emission from the sample edge is captured by the sighting optics of the spectrophotometer. The angle of observation with respect to the normal of the edge surface can be varied.

For local excitation, the spot of the UV excitation can be spatially limited by a slit (width $1 \mathrm{~mm}$ ). By lateral displacements of the excitation spot, the mean optical path length for lateral energy transport within the sample can be varied.

In order to measure spectra of the total hemispherical transmittance and reflectance, the spectrophotometer can be combined with an integrating sphere (LABSPHERE RT-060-SF/IG) and a suitable light source (150 W Xenon arc lamp). For measurements of the total hemispherical reflectance, a highly reflecting calibration standard (LABSPHERE SCS-99-020) has been used, with a total hemispherical reflectance above $98.8 \%$ for all wavelengths in the visible spectral range. The acquired raw data are corrected by the known reflectance of the reference sample. 


\section{Results}

From measurements of total hemispherical transmittance and reflectance, the spectral absorptance of the deposited coatings has been inferred. Fig. 2 illustrates the results for $\mathrm{CdS}$ containing silicon dioxide films $\left(\mathrm{SiO}_{2}: \mathrm{CdS}\right)$. In the representation, the spectral absorptance data are plotted as a function of the energy of the incident photons. For CdS-rich films (at $\% \mathrm{Cd}: a t \% \mathrm{Si}=0.84$ ) an absorption edge in the vicinity of the energy of the band gap of bulk $\mathrm{CdS}\left(E_{\mathrm{g}}=2.42 \mathrm{eV}\right)$ is observed. For lower Cd concentrations the onset of the absorption edge shifts monotonously to higher energies. The values observed in the range from $2.7 \mathrm{eV}$ to $2.9 \mathrm{eV}$ correspond in finite well and tight binding calculations (Nosaka, 1991; Lippens and Lannoo, 1989) approximately to cluster radii between $1 \mathrm{~nm}$ and $2 \mathrm{~nm}$.

The coatings exhibit visible photoluminescence, which is concentrated within the sample, and emitted from edges. PL spectra are obtained under broad band ultraviolet UVA illumination as a function of the emission angle (see Fig. 3a). The angle-dependence of the spectra is illustrated by Fig. 3b. For a $\mathrm{SiO}_{2}: \mathrm{CdS}$ sample annealed at $250^{\circ}$ (with at $\% \mathrm{Cd}: \mathrm{at} \% \mathrm{Si}=0.11$ ) two emission bands are observed, a dominant feature centered at $550 \mathrm{~nm}$, and a smaller contribution at $480 \mathrm{~nm}$. For an emission angle $\theta$ of $45^{\circ}$, the feature at $480 \mathrm{~nm}$ forms a shoulder located on one of the wings of the main peak. For decreasing angles the relative contribution of the band at $480 \mathrm{~nm}$ becomes more dominant $\left(\theta=30^{\circ}\right)$ and the shoulder turns into a peak $\left(\theta=15^{\circ}\right)$.

Photoluminescence spectra can also be obtained for a constant emission angle $\theta$ by exciting the sample locally and varying the spot of UV radiation, inducing different mean path lengths for the radiation laterally guided within

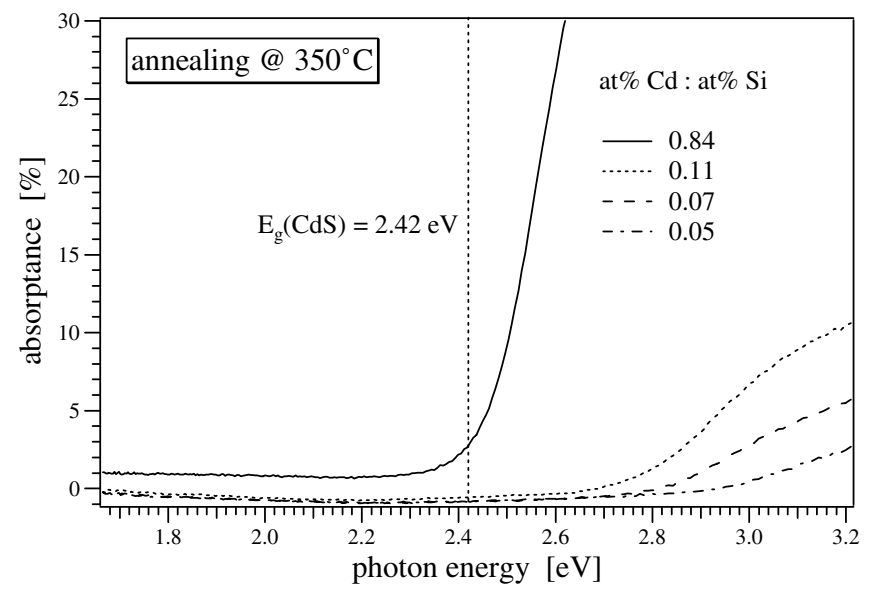

Fig. 2. Spectral absorptance of $\mathrm{SiO}_{2}: \mathrm{CdS}$ films on low iron glass, as determined by measurements of total hemispherical transmittance and reflectance. For CdS-rich films (at $\% \mathrm{Cd}: \mathrm{at} \% \mathrm{Si}=0.84)$, an absorption edge is observed for photon energies in the vicinity of the energy gap of bulk $\mathrm{CdS}\left(E_{\mathrm{g}}=2.42 \mathrm{eV}\right)$. For lower CdS contents, the absorption edge shifts monotonously to higher energies. From the blueshift, CdS cluster radii in the order of $1-2 \mathrm{~nm}$ can be estimated by comparison to finite potential well and tight binding calculations. a
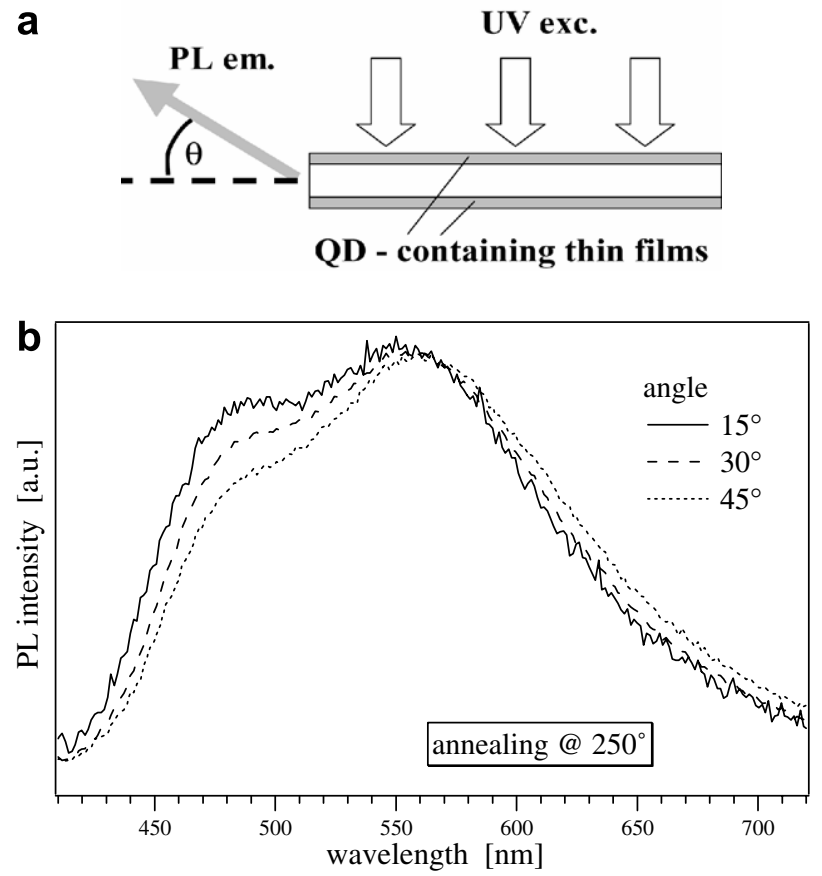

Fig. 3. (a) For measurements of photoluminescence spectra, the sample is illuminated by broad band UVA radiation. Spectra are obtained for different angles $\theta$ of emission from the edges. (b) Angle-dependent photoluminescence spectra obtained from a $\mathrm{SiO}_{2}: \mathrm{CdS}$ thin film on low iron glass. Two emission bands are observed, one at approx. $480 \mathrm{~nm}$ and a second band at approx. $550 \mathrm{~nm}$. The relative contribution of the band around $480 \mathrm{~nm}$ is higher for smaller emission angles $\theta$.

the sample (see Fig. 4a). Fig. 4b shows data for the same $\mathrm{SiO}_{2}$ :CdS sample described above. For the spot of UV excitation localized at a distance of $5 \mathrm{~mm}$ from the emitting edge, a peak is observed at wavelengths around $480 \mathrm{~nm}$. This feature becomes less pronounced for an increasing lateral path. The shape of the large emission band around $560 \mathrm{~nm}$ remains nearly unchanged regarding variations of the lateral path in the given range. The reabsorption of the PL emission in the spectral region around $480 \mathrm{~nm}$ is thus stronger than around $550 \mathrm{~nm}$. This observation can easily be explained by the onset of interband absorption (compare with Fig. 2).

Photoluminescence spectra obtained from a series of CdS quantum dot containing $\mathrm{SiO}_{2}$ samples are shown in Fig. 5. The only parameter varied has been the temperature of thermal annealing, the chemical composition (at $\%$ $\mathrm{Cd}: \mathrm{at} \% \mathrm{Si}=0.11$ ) has been kept constant. The emission angle $\theta$ was $30^{\circ}$, and the samples were excited globally with UVA radiation. For annealing at $450{ }^{\circ} \mathrm{C}$, the PL signal is dominated by a large band centered around $590 \mathrm{~nm}$. For annealing at $350{ }^{\circ} \mathrm{C}$, the dominating component shifts slightly to approx. $580 \mathrm{~nm}$, and a shoulder at approx. $490 \mathrm{~nm}$ appears. For annealing at $250^{\circ} \mathrm{C}$, the dominating peak shifts to approx. $560 \mathrm{~nm}$ while the component at shorter wavelengths becomes clearly distinguishable around $480 \mathrm{~nm}$.

A photograph of the $\mathrm{SiO}_{2}: \mathrm{CdS}$ samples formed at $250{ }^{\circ} \mathrm{C}, 350{ }^{\circ} \mathrm{C}$, and $450{ }^{\circ} \mathrm{C}$ is shown in Fig. 6 . The samples 
a
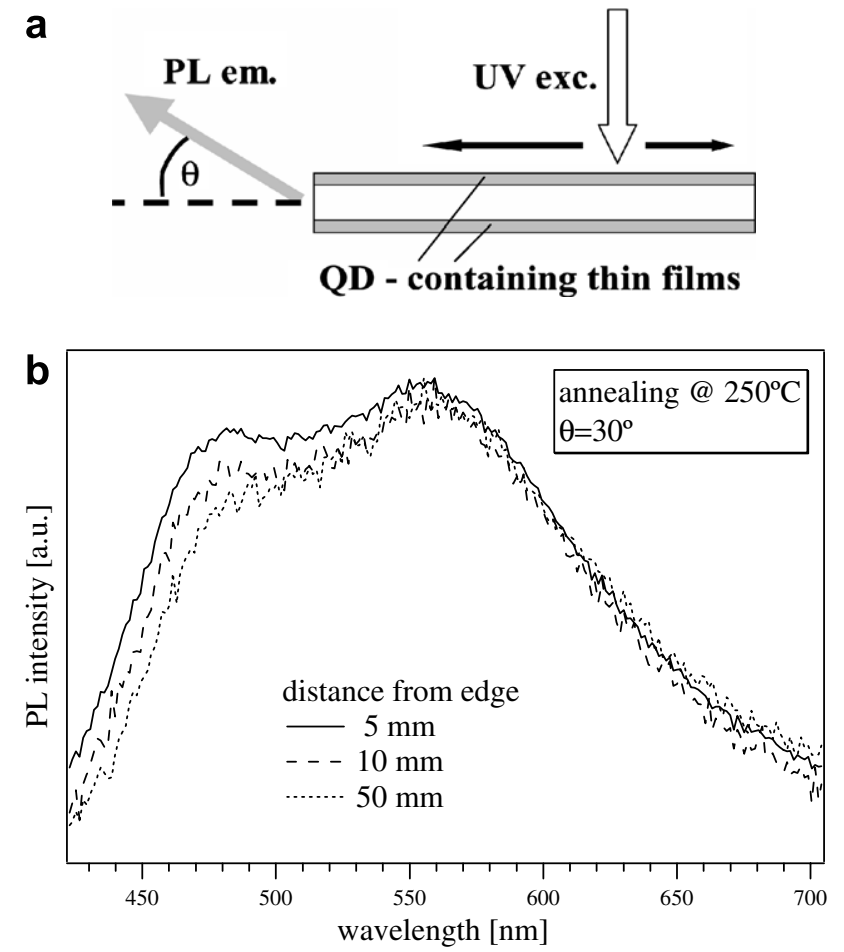

Fig. 4. (a) Local excitation of the sample by ultraviolet radiation. The optical path length for the radiation guided laterally within the sample can be varied simply by displacing the spot of excitation. (b) Photoluminescence spectra at an emission angle $\theta=30^{\circ}$ for spatial variation of the spot of localized UV excitation. Increasing the mean optical path of lateral energy transport within the sample results in a decrease of the relative contribution of the band at $480 \mathrm{~nm}$.

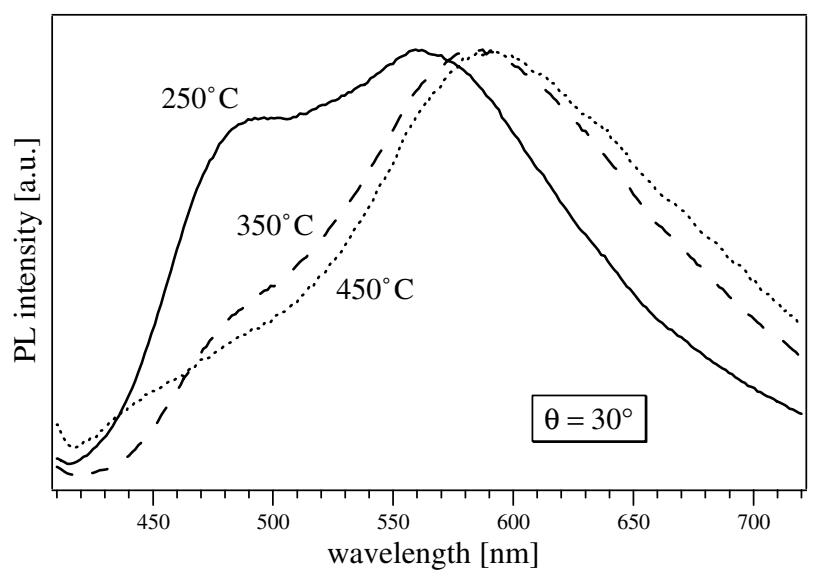

Fig. 5. Photoluminescence spectra obtained from a series of $\mathrm{SiO}_{2}: \mathrm{CdS}$ samples annealed at temperatures of $250{ }^{\circ} \mathrm{C}, 350^{\circ} \mathrm{C}$ and $450{ }^{\circ} \mathrm{C}$. For lower annealing temperatures, a blueshift of the dominating peak and the appearance of a higher wavelength component are observed. Since the annealing temperature is the only parameter which has been changed regarding the preparation of the samples, it can be assumed that the displayed spectra reveal quantum size effects.

are illuminated by UVA radiation. The strong emission from the edges of the samples is due to the concentration of the photoluminescent radiation in the waveguides by

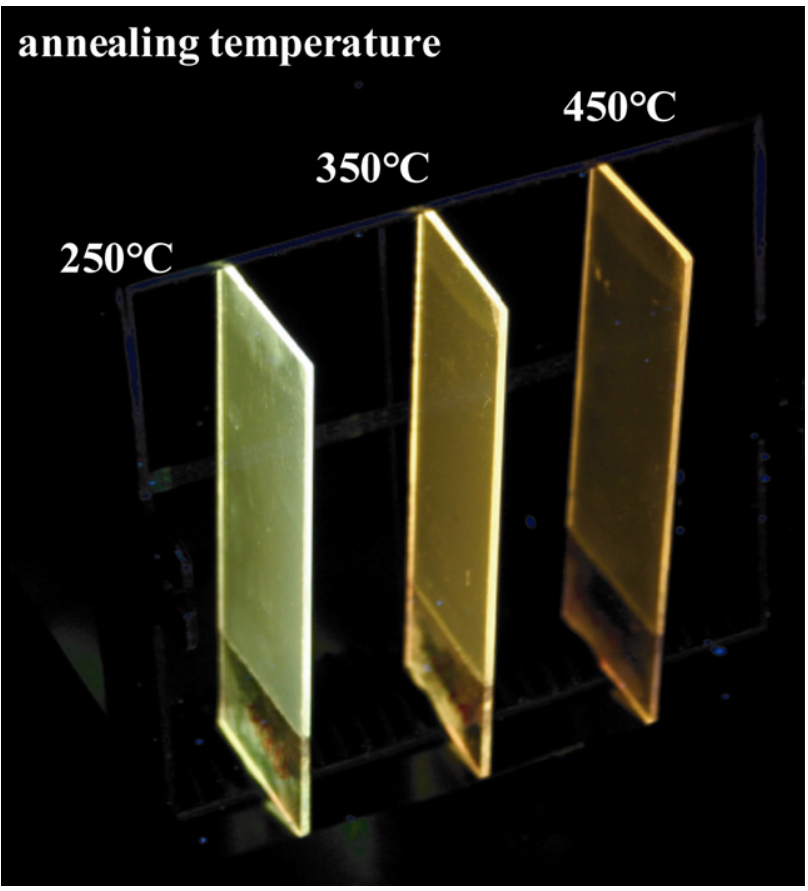

Fig. 6. Photograph of the $\mathrm{SiO}_{2}$ : $\mathrm{CdS}$ samples formed at $250{ }^{\circ} \mathrm{C}, 350{ }^{\circ} \mathrm{C}$, and $450^{\circ} \mathrm{C}$. The strong emission from the edges of the samples is due to the concentration of the photoluminescent radiation in the waveguides by total internal reflection. The obtained colors of the visible photoluminescence, ranging from green for $250^{\circ} \mathrm{C}$ to yellow for $350{ }^{\circ} \mathrm{C}$ and orange for $450^{\circ} \mathrm{C}$, illustrate the effects of quantum confinement.

total internal reflection at the surface of the coating. The obtained colors of the visible photoluminescence range from green for $250^{\circ}$ to yellow for $350^{\circ}$ and orange for $450^{\circ}$. Since the cluster growth during the thermal processing step is governed by the annealing temperature, it is straightforward to assume that for higher temperatures larger clusters are formed. The different colors in the photograph (Fig. 6) and the spectra shown in Fig. 5 reflect thus directly the effects of quantum confinement.

\section{Discussion}

It has been shown that anorganic photoluminescent coatings can be produced by the sol-gel dip-coating process. The optical data of as well the spectral absorptance as well as the photoluminescence strongly suggest that we have succeeded in synthesizing $\mathrm{CdS}$ nanocrystal containing $\mathrm{SiO}_{2}$ films. For $\mathrm{SiO}_{2}: \mathrm{CdS}$ films with a $\mathrm{CdS}$ volume fraction above the percolation threshold, an absorption edge at photon energies close to the value of bulk $\mathrm{CdS}$ would be expected. The blueshift of the absorption edge observed for low $\mathrm{CdS}$ volume fractions can be explained by quantum confinement and suggests the formation of nanometersized CdS crystallites. A finite potential well model (Nosaka, 1991) and results from tight binding calculations (Lippens and Lannoo, 1989) have been used to estimate the mean cluster radii to be in the range of $1-2 \mathrm{~nm}$. The photoluminescent properties of the coatings are developed 
during the thermal annealing step. It can thus be inferred that the growth of the nanocrystals takes place during thermal annealing. The temperature of annealing plays a crucial role in the crystal growth progress, and it seems obvious that higher annealing temperatures result in an increasing mean cluster size. The effects of quantum confinement are thus expected to be stronger for samples produced at lower temperatures.

The results from spectral photoluminescence measurements confirm the hypothesis of the presence of CdS nanocrystals in such films. The characteristical two-band structure of the emission spectra has also been observed by other researchers for CdS nanocrystals immersed in silica matrices (Capoen et al., 2001; De la Rosa-Fox et al., 2003). Capoen et al. designated the high- and low-energy components of the PL emission by the labels "band I" and "band II", respectively, and explained the former by the direct recombination of the exciton pairs while ascribing the latter to a recombination of the exciton pairs via trap surface states. The underlying model (Hässelbarth et al., 1993) includes two surface trap bands associated with interstitial S (Pedrotti and Reynolds, 1960), and excess Cd (Wang and Herron, 1988). Due to the non-radiative relaxation of electronic states (hole relaxation to excess $\mathrm{Cd}$ surface trap band), some energy is lost in the cycle of excitation and band II emission. However, as shown by the measurements, the large Stokes shift for band II results in a lower reabsorption. The transversal type of measurement as illustrated by Fig. 4, where the optical path within the quantum dot containing material is relatively long, is much more sensitive to weak absorption than the determination of spectral absorptance by measuring normal transmittance and reflectance (see Fig. 2). Since the reabsorption is the main limiting factor for the concentration, this effect is also beneficial because it implies the possibility of higher concentration factors. It is interesting to note that not only the emission related to direct recombination (band I) but also the emission related to surface states (band II) shift to higher energies for samples annealed at lower temperatures. Quantum confinement has obviously an effect also on the electronic states involved in band II emission. For samples annealed at lower temperatures, the relative contribution of the emission attributed to direct recombination increases, which might be understood in terms of the growing oscillator strength for increasing quantum confinement due to the increasing spatial overlap of electron and hole wavefunctions.

Preliminary investigations of similarly prepared $\mathrm{SiO}_{2}$ : $\mathrm{TiO}_{2}$ films by means of transmission electron microscopy have shown evidence for a granular structure with grain sizes in the order of several nanometers. A structure model based on cadmium sulfide nanorystallites immersed in silicon dioxide appears thus as reasonable for ternary $\mathrm{Si}-\mathrm{O}$ $\mathrm{Cd}-\mathrm{S}$ films. However, a detailed characterization by complementary experimental methods such as e.g. transmission electron microscopy and X-ray diffraction would be necessary to exclude the formation of ternary and quaternary phases and to confirm the presence of nanocrystalline CdS.
The sol-gel process has a large potential for large area deposition at low cost and is considered as promising also in other fields of solar energy conversion, concerning optical selective absorber coatings (Boström et al., 2003), and thin films on thermal collector glazing (Granqvist, 1998; Gombert et al., 2000). Therefore, the proposed concept of anorganic thin film photoluminescent solar concentrators could be very promising regarding the criterion of energy cost. The use of quantum dots provides stability against photobleaching under solar irradiation as well as a great flexibility in emission wavelength due to the beneficial effects of quantum confinement. Additionally, there is some hope that the purity of the material is less critical than for photovoltaic cells, where electron transport has to be assured everywhere in the semiconductor materials. This could imply a reduction in "grey" energy involved in the production process. Finally, the proposed collectors based on coated glass panes might be very suitable for architectural integration into the building envelope. In order to estimate the efficiency and possible concentration ratios for such devices, detailed optical characterizations of the coating materials are still necessary. The choice of environmental harmless constituents and the optimization of their photoluminescent properties belong to the major challenges for future work.

\section{Conclusions}

Quantum dot containing nanocomposite coatings might be an interesting alternative for the production of planar quantum dot solar concentrators. By sol-gel dip-coating, photoluminescent anorganic thin films have been deposited on low iron glass substrates. The optical data on absorption and photoluminescence strongly suggest that the synthesis of cadmium sulfide nanocrystallite containing silicon dioxide films $\mathrm{SiO}_{2}: \mathrm{CdS}$ has succeeded. The blueshift of the absorption edge observed for low $\mathrm{Cd}$ concentrations directly reflects the effect of quantum confinement. A finite potential well model and results from tight binding calculations can be used to estimate the mean cluster radii to be in the range of 1-2 $\mathrm{nm}$. In the photoluminescence spectra, two emission bands are observed, which are associated with direct recombination and recombination via trap surface states, respectively. For lower annealing temperatures, a blueshift of the occurring features in the PL spectra is observed and associated with the increasing quantum confinement due to shrinking cluster size. As seen from angledependent emission spectra and for variation of the optical path length in the sample, the emission band at lower photon energies propagates more easily in the waveguide than the high-energy band. The lower reabsorption is related to the larger Stokes shift and could be advantageous for achieving higher concentration factors. Given the suitability of thin film technology for large area deposition at low cost, quantum dot containing nanocomposite coatings on highly transparent substrates can be considered as an inter- 
esting alternative for the fabrication of photoluminescent planar solar concentrators.

\section{Acknowledgements}

Financial support of this work has been provided by the Swiss Federal Office of Energy SFOE. Authors are grateful to C. Roecker and P. Loesch for technical support, to L. Deschamps for software development, and to C. Galande for photography.

\section{References}

Barnham, J., Marques, J.L., Hassard, J., O’Brien, P., 2000. Quantum-dot concentrator and thermodynamic model for the global redshift. Appl. Phys. Lett. 76, 1197-1199.

Boström, T., Wäckelgard, E., Westin, G., 2003. Solution-chemical derived nickel-alumina coatings for thermal solar absorbers. Sol. Energy 74, 497-503.

Brus, L.E., 1983. A simple model for the ionization potential, electron affinity and aqueous redox potentials of small semiconductor crystallites. J. Chem. Phys. 79, 5566-5571.

Capoen, B., Gacoin, T., Nédélec, J.M., Turrell, S., Bouazaoui, M., 2001. Spectroscopic investigations of CdS nanoparticles in sol-gel derived polymeric thin films and bulk silica matrices. J. Mater. Sci. 36, 25652570 .

Crouch, D., Norager, S., O’Brien, P., Park, J.-H., Pickett, N., 2003. New synthesis routes for quantum dots. Phil. Trans. R. Soc. Lond. A 361, 297-310.

Darugar, Q., Qian, W., El-Sayed, M.A., 2006. Observation of optical gain in solutions of CdS quantum dots at room temperature in the blue region. Appl. Phys. Lett. 88, 261108-1-261108-3.

De la Rosa-Fox, N., Piñero, M., Litrán, R., Esquivias, L., 2003. Photoluminescence from CdS quantum dots in silica gel. J. Sol-Gel Sci. Technol. 26, 947-951.

De Mello Donegá, C., Hickey, S.G., Wuister, S.F., Vanmaeckelbergh, D., Meijerink, A., 2003. Single-step synthesis to control the photoluminescence quantum yield and size dispersion of CdSe nanocrystals. J. Phys. Chem. 107, 489-496.

Éfros, Al.L., Éfros, A.L., 1982. Interband absorption of light in a semiconductor sphere. Sov. Phys. Semicond. 16, 772-775.

Gallagher, S.J., Eames, P.C., Norton, B., 2004. Quantum dot solar concentrator, predicted using a ray trace approach. Int. J. Appl. Energy 25, 47-56.

Goetzberger, A., Greubel, W., 1977. Solar energy conversion with fluorescent collectors. Appl. Phys. 14, 123-139.

Gombert, A., Glaubitt, W., Rose, K., Dreibholz, J., Bläsi, B., Heinzel, A., Sporn, D., Doll, W., Wittwer, V., 2000. Antireflective transparent covers for solar devices. Sol. Energy 68, 357-360.

Granqvist, C.G., 1998. Progress in solar energy materials: examples of work at Uppsala University. Renew. Energy 15, 243-250.

Greenham, N.C., Peng, X., Alivisatos, A.P., 1996. Charge separation and transport in conjugated-polymer/semiconductor-nanocrystal composites studied by photoluminescence quenching and photoconductivity. Phys. Rev. B 54, 17628-17637.

Hässelbarth, A., Eychmüller, A., Weller, H., 1993. Detection of shallow electron traps in quantum-sized $\mathrm{CdS}$ by fluorescence quenching experiments. Chem. Phys. Lett. 203, 271-276.

Hines, M.A., Guyot-Sionnest, P., 1996. Synthesis and characterization of strongly luminescing ZnS-capped CdSe nanocrystals. J. Phys. Chem. $100,468-471$.

Hu, Y.Z., Koch, S.W., Lindberg, M., Peyghambarian, N., Pollock, E.L., Abraham, F.F., 1990. Biexcitons in semiconductor quantum dots. Phys. Rev. Lett. 64, 1805-1807.
Ishikawa, T., Nishimura, T., Kohmoto, S., Asakawa, K., 2000. Sitecontrolled InAs single quantum-dot structures on GaAs surfaces patterned by in situ electron-beam lithography. Appl. Phys. Lett. 76, $167-169$.

Kang, K.I., McGinnis, B.P., Sandalphon, Hu, Y.Z., Koch, S.W., Peyghambarian, N., Mysyrowicz, A., Liu, L.C., Risbud, S.H., 1992. Confinement-induced valence-band mixing in CdS quantum dots observed by two-photon spectroscopy. Phys. Rev. B 45, 3465-3468.

Kayanuma, Y., 1988. Quantum-size effects of interacting electrons and holes in semiconductor microcrystals with spherical shape. Phys. Rev. B 38, 9797-9805.

Lee, S., Shin, D.W., Kim, W.M., Cheong, B., Lee, T.S., Lee, K.S., Cho, S., 2006. Room temperature synthesized GaAs quantum dot embedded in $\mathrm{SiO}_{2}$ composite film. Thin Solid Films 514, 296-301.

Lifshitz, E., Dag, I., Litvin, I., Hodes, G., Gorer, S., Reisfeld, R., Zelner, M., Minti, H., 1998. Optical properties of CdSe nanoparticle films prepared by chemical deposition and sol-gel methods. Chem. Phys. Lett. 288, 188-196.

Lippens, P.E., Lannoo, M., 1989. Calculation of the band gap for small CdS and ZnS crystallites. Phys. Rev. B 39, 10935-10942.

Luque, A., Martí, A., 1997. Increasing the efficiency of ideal solar cells by photon induced transitions at intermediate levels. Phys. Rev. Lett. 78, 5014-5017.

Murray, C.B., Norris, D.J., Bawendi, M.G., 1993. Synthesis and characterization of nearly monodisperse $\mathrm{CdE}(\mathrm{E}=\mathrm{S}, \mathrm{Se}, \mathrm{Te})$ semiconductor nanocrystallites. J. Am. Chem. Soc. 115, 8706-8715.

Nirmal, M., Dabbousi, B.O., Bawendi, M.G., Macklin, J.J., Trautman, J.K., Harris, T.D., Brus, L.E., 1996. Fluorescence intermittency in single cadmium selenide nanocrystals. Nature 383, 802-804.

Nosaka, Y., 1991. Finite depth spherical well model for excited states of ultrasmall semiconductor particles. An application. J. Phys. Chem. 95, 5054-5058.

Nozik, A.J., 2002. Quantum dot solar cells. Physica E 14, 115-120.

Oelhafen, P., Schüler, A., 2005. Nanostructured materials for solar energy conversion. Sol. Energy 79, 110.

Parvathy, N.N., Pajonk, G.M., Venkateswara Rao, A., 1997. Synthesis and study of quantum size effect, XRD and IR spectral properties of $\mathrm{PbS}$ nanocrystals doped in $\mathrm{SiO}_{2}$ xerogel matrix. J. Cryst. Growth 179, 249-257.

Pedrotti, L.S., Reynolds, D.C., 1960. Energy model for edge emission in cadmium sulfide. Phys. Rev. B 120, 1664-1669.

Plass, R., Pelet, S., Krueger, J., Grätzel, M., Bach, U., 2002. Quantum dot sensitization of organic-inorganic hybrid solar cells. J. Phys. Chem. B 106, 7578-7580.

Schüler, A., Gampp, R., Oelhafen, P., 1999. In situ photoelectron spectroscopy of titanium-containing amorphous hydrogenated carbon films. Phys. Rev. B 60, 16164-16169.

Schüler, A., Videnovic, I.R., Oelhafen, P., Brunold, S., 2001. Titaniumcontaining amorphous hydrogenated silicon carbon films (a-Si:C:H/ Ti) for durable solar absorber coatings. Sol. Energy Mater. Sol. Cell 69, 271-284.

Tasco, V., Todaro, M.T., De Vittorio, M., De Giorgi, M., Cingolani, R., Passaseo, P., Ratajczak, J., Katcki, J.W., 2004. Electrically injected InGaAs/GaAs quantum dot microcavity light-emitting diode operating at $1.3 \mu \mathrm{m}$ and grown by metalorganic chemical vapor deposition. Appl. Phys. Lett. 84, 4155-4157.

Van Sark, W.G.J.H.M., Meijerink, A., Schropp, R.E.I., van Roosmalen, J.A.M., Lysen, E.H., 2005. Enhancing solar cell efficiency by using spectral converters. Sol. Energy Mater. Sol. Cell 87, 395-409.

Wang, Y., Herron, N., 1988. Photoluminescence and relaxation dynamics of CdS superclusters in zeolites. J. Phys. Chem. 92, 4988-4994.

Wang, Y., Herron, N., 1990. Quantum size effects on the exciton energy of CdS clusters. Phys. Rev. B 42, 7253-7255.

Wijayantha, K.G.U., Peter, L.M., Otley, L.C., 2004. Fabrication of CdS quantum dot sensitized solar cells via a pressing route. Sol. Energy Mater. Sol. Cells 83, 363-369. 